\title{
Recovery and Visualization of 3D Structure of Chromosomes from Tomographic Reconstruction Images
}

\author{
Sabarish Babu, ${ }^{1}$ Pao-Chuan Liao, ${ }^{1}$ Min C. Shin, ${ }^{1}$ and Leonid V. Tsap ${ }^{2}$ \\ ${ }^{1}$ Department of Computer Science, University of North Carolina at Charlotte, 9201 University City Boulevard, \\ Charlotte, NC 28223, USA \\ ${ }^{2}$ Systems Research Group, Electronics Engineering Department, University of California Lawrence Livermore National Laboratory, \\ Livermore, CA 94551, USA
}

Received 27 April 2005; Revised 12 October 2005; Accepted 21 December 2005

The objectives of this work include automatic recovery and visualization of a 3D chromosome structure from a sequence of $2 \mathrm{D}$ tomographic reconstruction images taken through the nucleus of a cell. Structure is very important for biologists as it affects chromosome functions, behavior of the cell, and its state. Analysis of chromosome structure is significant in the detection of diseases, identification of chromosomal abnormalities, study of DNA structural conformation, in-depth study of chromosomal surface morphology, observation of in vivo behavior of the chromosomes over time, and in monitoring environmental gene mutations. The methodology incorporates thresholding based on a histogram analysis with a polyline splitting algorithm, contour extraction via active contours, and detection of the $3 \mathrm{D}$ chromosome structure by establishing corresponding regions throughout the slices. Visualization using point cloud meshing generates a 3D surface. The 3D triangular mesh of the chromosomes provides surface detail and allows a user to interactively analyze chromosomes using visualization software.

Copyright () 2006 Hindawi Publishing Corporation. All rights reserved.

\section{INTRODUCTION}

\subsection{Motivation}

Tracking and visualizing chromosomes gives biologists valuable information regarding their three-dimensional (3D) structure and behavior. Previously, segmentation of banded chromosomes frozen in metaphase of mitosis was important for classification especially in the karyotyping process. This process facilitates the classification and detection of chromosomal abnormalities such as Klinefelter's, Down's, and Turner's syndrome. Chromosome analysis is important in such situations as prenatal amniocentesis examination, detection of malignant diseases, and monitoring environmental gene mutations.

In this paper, we propose a new method for (1) an automatic recovery of chromosomes in a sequence of $2 \mathrm{D}$ fluorescence volume image slices, and (2) visualizing the resulting chromosomes in $3 \mathrm{D}$. Such $3 \mathrm{D}$ visualization provides biologists with information that cannot be obtained by $2 \mathrm{D}$ images alone. Commonly used imaging systems rely on reconstruction of an image from its projections through the process of computed tomography (CT) which generates fluorescent optical sections also known as volume image slices.
In medical imaging, for example, X-ray plates, CT scans, magnetic resonance imaging (MRI), and various types of positron emission tomography (PET) all record 2D projections of 3D objects [1]. Hence, tracking the contour of an object along each successive slice allows us to recreate a $3 \mathrm{D}$ representation of the object. We see that $3 \mathrm{D}$ visualization of the chromosome can be useful for biologists in the following ways: (1) identifying the space occupied by the chromosome within the cell, (2) visualizing specific structures along the contour such as "constrict points," and binding sites with other intercellular molecules such as proteins (i.e., matrix-binding proteins which anchor the chromosome to the nucleus), enzymes, and other organelles, (3) using the visualization to accurately classify the chromosomes, (4) detecting anomalies, such as chromosomal disorders, (5) helping to identify and observe the behavior of the organelle over time (sometimes called $4 \mathrm{D}$ reconstruction, with time as the fourth dimension), especially during cellular expression and replication, (6) analysing genomic mutations such as deletions and inversions, and (7) studying DNA conformation (packaging) within the chromosome including the presence of DNA structural motifs, understanding how conformation is affected over time, and gene expression patterns. 


\subsection{Previous work}

Previous research of chromosomes in 2D images was primarily focused on abnormality detection and classification of chromosomes. In chromosome classification (Karyotyping), one of the main efforts includes the problem of separation of partially occluded chromosomes. Lerner et al. [2] proposed classification based on skeleton points, and local feature extraction for classification purposes (CPOOS_classificationdriven partially occluded object segmentation method). Shi et al. also used local features such as cut points, skeleton points, junction points, and ravine points to separate touching chromosomes using parallel mesh algorithm [3]. Some of the local features extracted were based on topology, such as concavities, that indicated where occlusions between chromosomes occurred. Hence, their separation and classification were based on landmarks and occlusion points. Lerner et al. [4] trained multilayer perceptron (MLP) neural networks to classify chromosomes and used a "knockout" technique as well as principle component analysis (PCA) for feature selection. Both techniques yielded the benefit of using only about $70 \%$ of the available features to get the most out of classifier performance. Vidal and Castro used syntactic/structural pattern recognition algorithms such as errorcorrecting grammatical interface (ECGI) and MLP to classify chromosomes by formulating rule-based string representation of the features extracted [5]. Keller et al. presented a fuzzy logic system in addition to neural network-based classification system to deal with ambiguities during the classification process. These ambiguities included imprecisions in computation, and in-class definitions in mid-level computer vision processes [6]. Minor chromosomal abnormalities cannot be detected by applying available techniques to $2 \mathrm{D}$ images. Based on banding patterns and skeletal line lengths, only major chromosomal abnormalities, such as deletions, can be detected. Inversion abnormalities may cause problems in information extraction for the formulation of rules in the syntactic/structural methods. However, 3D visualization enables scientists to discern occluding chromosomes for further classification better than 2D image analysis. Imelinska et al. proposed a semiautomatic region-based color segmentation algorithm to extract anatomic structures. Their basic approach included subdividing an image into regions inside or outside the target structure using Delaney triangulation, and then breaking up the regions on the boundary between the two classifications into smaller regions, and finally repeating the classification based on user input [7]. Holden et al. proposed a methodology for segmentation of brain lesions from MR images. Their segmentation algorithm consisted of contour detection followed by Haslett's contextual classification method extended to 3D [8]. Yan et al. described a semiautomatic method for segmentation of lymph nodes in CT images using the level set method [9]. Noordmans and Smeulders proposed a strategy that detects and characterizes isolated and overlapping spots in images, where spots are defined as image details without inner structure [10]. To apply the strategy to our domain, one would have to define a substantial, nontrivial set of spot models to suit our image data.
The proposed method requires a seed point and a circle to define an approximate region of interest for the level set operator to work on segmenting the biological object. Our region segmentation step employed within our automatic methodology uses polyline splitting algorithm to model the histogram contour, and was designed to simply and efficiently identify slices that could consist of regions corresponding to foreground chromosome and to subsequently select the appropriate threshold for region segmentation automatically.

There have also been several papers published on visualization and $3 \mathrm{D}$ reconstruction of large and small biological objects based on various imaging modalities. Volume rendering is the process of generating a $3 \mathrm{D}$ organ from $2 \mathrm{D}$ image slices. There are two methods of volume visualization: surface rendering which requires a preprocessing segmentation procedure and volume rendering where the reconstructed organ is directly generated from the original image slices. Although rendering effects of volume rendering provides the greatest amount of detail, volume rendering is slow. Surface rendering allows for easier manipulation and interaction with the biological object in 3D [11]. For some types of biological visualization, very high-resolution details of the surface structure may not be necessary. In the case of chromosome structural analysis, biologists are looking for a method of detecting abnormalities and other higher-level surface artifacts which do not require very detailed visualizations, hence in this case medium-resolution surface rendering would be sufficient. Chemical analysis of the DNA within the chromosome can reveal small-scale abnormalities and inconsistencies better. The visible human project of the National Library of Medicine used transverse CT, MR, and cryosection images of representative male and female cadavers to obtain 3D human body representations [12]. The cryosection images, used for full body visualization, were taken at regular intervals, the male was sectioned at onemillimeter intervals, and the female at one-third of a millimeter intervals. Subramanian et al. used intravascular ultrasound (IVUS) images, which is a technology for imaging the vascular lumen and atherosclerotic plague structure, and devised a technique to accurately reconstruct $3 \mathrm{D}$ geometry of blood vessels [13]. Various imaging modalities were also employed in 3D reconstruction such as biplane $\mathrm{X}$-ray fluoroscopy, X-ray, and echo images. The path of the catheter tip was estimated by fitting an interpolating spline through the $3 \mathrm{D}$ points. Arnison et al. presented a modality called differential interference contrast (DIC) microscopy and applied Hilbert transforms to distinguish features of chromosomes from background in each 2D slice, and selective opacity to $3 \mathrm{D}$ pixels (voxels) according to their intensity to visualize chromosomes [14]. Engelhardt et al. visualized metaphase chromosome from human (HeLa) cell lines using electron microscopy (EM) [15]. The images were aligned using colloidal gold particles as reference points, and reconstruction was produced by the weighted back-projection method. Liu et al. proposed a methodology to visualize and quantify brain tumor lesions from MRI volumetric images towards routine clinical evaluation of brain tumor patients [16]. They used a fuzzy connectedness framework for tumor segmentation, 
which requires some user intervention, towards detecting the tumor regions in 3D. Zoroofi et al. provided an automatic methodology for segmentation and $3 \mathrm{D}$ visualization of the diseased femoral head from 3D MR volumetric data [17]. Both segmentation of the femur and necrotic lesion classification were done in 3D. Viergever et al. have used an integrated multimodal approach towards segmentation, integration, and visualization of brain slices [18]. The volumetric data was acquired and integrated from CT, MRI, and SPECT input modalities. Qingsong et al. proposed a visualization approach that used surface as well as volume rendering techniques to visualize the human head towards surgical planning applications [11]. Since either integrating volume rendering methods or integrating volumetric data from several modalities can be slow, there have been efforts in rendering real-time visualizations of biological organs using commercial graphics hardware. For example, Levin et al. proposed a method of real-time visualization of a $4 \mathrm{D}$ volume visualization of a beating heart using the graphics processing unit [19].

Most techniques for the segmentation and visualization of 3D biological structures have been proposed for large biological organelles such as brain, heart, and bone tissues. Methods for the segmentation and 3D recovery of small intracellular organelles such as chromosomes, mitochondria, and endoplasmic reticulum are challenging to develop as these biological organelles are extremely small, and images containing volumetric data of such organelles consist of higher levels of noise. Until recently computer tomography (CT), which has been regarded as a fastest, most-detailed, and highest-resolution detection technology for in vivo biological structures, was unable to detect and produce volumetric data of very small objects such as intracellular structures. With the advent of new and improved techniques in computer tomography, it now becomes possible to quickly and accurately reconstruct $2 \mathrm{D}$ volumetric slices of minute structures such as intracellular organelles in their native state [20]. Our work focuses on a methodology for automatic recovery and visualization of chromosomes in tomographic reconstruction (CT) volume image slices. Our visualization approach also employs surface rendering as opposed to volume rendering as surface geometry is reconstructed from the image data. Our methodology can also be extended to recover and visualize other intracellular organelles such as mitochondria and human chromosomes, which are also of great interest to biologists when such data becomes available. In following sections, we explain in detail the various steps of our methodology and show results of the visualizations of the reconstructed Drosophila chromosomes.

\section{METHODOLOGY}

\subsection{Overview of the approach}

The objective of our research is to track the contour of chromosomes in a sequence of tomographic reconstruction images, thus enabling us to recover the chromosome object and to provide visualization. Images generated through tomographic fluorescence data are a form of commonly used fluorescence-based technique to generate medical volume image slices. The dataset was generated in the Sedat Lab at the University of California San Francisco [20]. The slices are grayscale images of two chromosomes of the common fruit fly (Drosophila melanogaster). The slices progress along a plane of capture, and total sixty-five slice images at $478 \times$ 512 resolution. The images consist of relatively high contrast and each slice is contaminated with many reconstruction artifacts.

Our proposed methodology consists of five stages.

(1) Segmentation of the chromosome regions in each 2D image slice is performed by image thresholding. The threshold is automatically selected by analyzing the histogram contour using a polyline splitting algorithm [21].

(2) Noise removal is achieved by connected component labeling (CCL) [21] to filter out foreground regions below a certain size.

(3) Two-dimensional contour refinement on each slice is performed on the contour of the chromosome regions extracted after step 2. This step employs an active contour model (snake) technique [22].

(4) Region correspondence is performed by corresponding the $2 \mathrm{D}$ regions of the same chromosome in adjacent slices. We use a region comparison method proposed by Hoover et al. [23] to correspond regions of the same chromosome between slices. This method achieves correspondence even when the chromosome breaks into multiple regions in some slices.

(5) Visualization of the chromosome in 3D consists of two steps. Initially, we extract a set of nodes from the contour of a single chromosome in each slice using chaincoding algorithm [21]. This set is taken for each chromosome, to create point clouds. Then, using meshing technique [24], we construct a mesh representing the surface of each chromosome.

These steps are described in separate sections in this paper. Figure 1 illustrates the flow chart of our methodology, showing sample output images from each step described above.

\subsection{Dataset}

Various imaging systems rely on reconstruction of an image from its projections through the process of computed tomography (CT). In medical imaging, for example, X-ray plates, CT scans, magnetic resonance imaging (MRI), and various types of positron emission tomography (PET) all record 2D projections of 3D objects [1]. Our dataset consists of tomographic reconstruction of chromosome volume image slices through the cell of a fruit fly (Drosophila melanogaster). There are a total of 65 images representing slices taken along a plane of capture. Most medical imaging systems separately reconstruct $2 \mathrm{D}$ slices of a $3 \mathrm{D}$ object. Those slices closest to Slice 1 are more indistinct, consisting primarily of background. Some of the slices, in particular ones closer to the end of the sequence, contain significant noise, which makes the task of chromosome segmentation more difficult. 

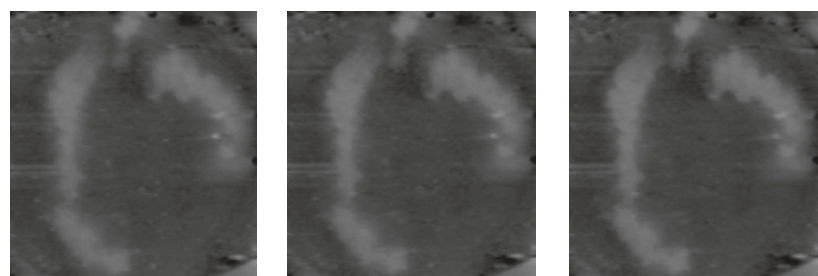

(a) Input images: the three sample input images on the right are from the set of tomographic reconstruction volume images. The total number of slices is 65 , and is greyscale. From left to right, the image samples are slice number 23,24 , and 25 , respectively
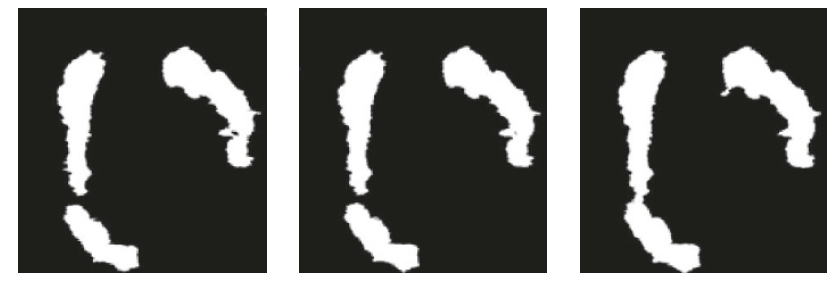

(c) Noise removal: images on the right show the result of noise removal performed on the output images of region segmentation
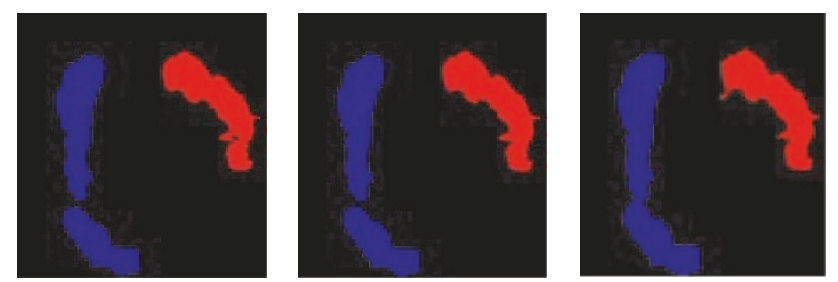

(e) Region correspondence: finding respective chromosomes in adjacent slides yields regions on left corresponded to chromosome 1 and right to chromosome 2
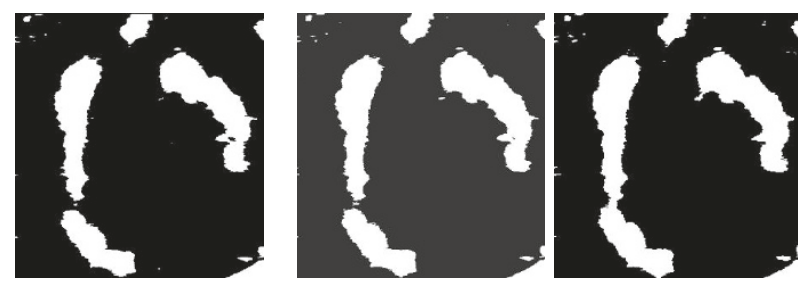

(b) Region segmentation: sample binary images from left to right are output images after region segmentation has been performed
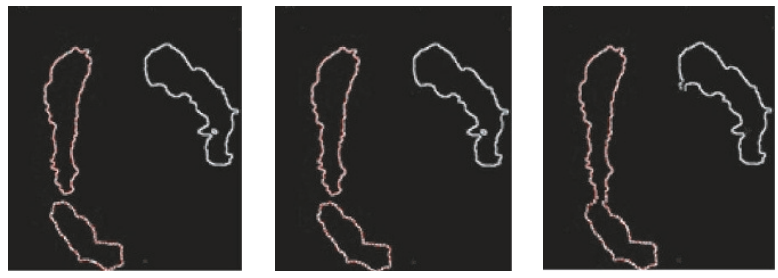

(d) 2D contour refinement: using the resulting contour as an initial estimate, we apply active contour models (snakes) [22] to refine it. Image on the right shows the refined contour of chromosome 1 (left) in slices 23,24 , and 25 , respectively

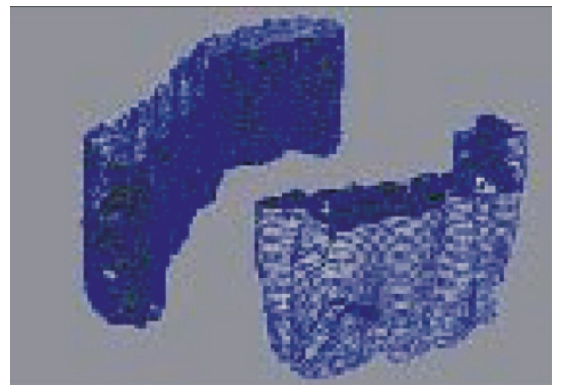

(f) Visualization: points along the refined contour corresponding to each chromosome (nodes) are extracted to obtain point clouds. Meshing technique [24] is applied to the point clouds to obtain the mesh describing the surface of the chromosome

FIGURE 1: Flow chart of the methodology showing sample input images, and output images corresponding to steps outline in the left column.

\section{REGION SEGMENTATION AND NOISE REMOVAL}

\subsection{Overview}

The goal of this step is to create a methodology that allows us to detect the presence of foreground regions that may consist of chromosomes, and to segment chromosome region from its background in each $2 \mathrm{D}$ slice, through automatic selection of an appropriate threshold for segmentation. In this step, we also remove slices for which suitable thresholds could not be determined since those slices only contain background. The histograms of the slices are either unimodal or multimodal. The histograms of the top-end and bottom-end slices are unimodal, as those slices contain background only. The histograms of the middle slices, however, are multimodal. To perform thresholding and subsequently segment chromosomes from the background successfully, we must establish that the histograms of the slices are multimodal.

Segmentation of many clinical and biological images is currently performed using manual slice editing [25]. This method has some deficiencies, such as difficulty in achieving reproducible results, operator bias, and it is tedious to perform. Segmentation using techniques, such as region growing, edge detection, and mathematical morphology operations, mostly requires considerable amounts of expert interactive guidance because some knowledge of the domain (the content of images) is necessary. Hence, automatic segmentation with little to no human intervention would be preferred. Otsu's method of thresholding (recommended by Shi et al. [3]), based on minimizing intragroup variance and maximizing intergroup variance, is not applicable to this dataset. These algorithms assume that the histogram is bimodal and demonstrates essentially two distributions. 
Hence, we seek an automatic method that analyses the histogram contour of each slice to determine whether it is unimodal or multimodal, excludes unimodal slices, and selects an appropriate threshold for segmentation. Wilcoxon's ranksum test [26], which is similar to a paired $T$-test for nonparametric data, could also be used as a criterion for determining whether the foreground is significantly different from background from two sets of data. Our segmentation step differs from previous methods by automatically processing in single step, excluding slices that do not contain chromosome and finding the appropriate threshold for segmentation by simply and effectively analyzing the histogram contour.

Applying polyline splitting to model the contour of the histogram enables us to determine whether the histogram is unimodal or multimodal, subsequently excluding slices with a unimodal histogram. This method also provides a basis for threshold selection through a reasonable measure such as the peakiness method [21], which will be described in the following section. Details of the polyline splitting algorithm can be found in [21]. By using the polyline splitting algorithm on a histogram contour, we can find the list of edges with vertices end to end that describes the histogram curve by recursively splitting it into line segments.

Slice 2 (Figure 2(a)), one of the top-end slices, is unimodal (Figure 2(c)) and it is therefore very difficult to distinguish foreground from background. In contrast, slice 35 (Figure 2(b)), one of the images from the middle slices, is bimodal (Figure 2(d)). Hence, it is possible to extract the chromosome from background using region segmentation.

\subsection{Description of the process}

The goal of this step is to robustly segment chromosome regions by (1) determining whether the image contains any chromosome and (2) finding the correct threshold even when the histogram of image contains multiple modalities. The polyline splitting algorithm is used to analyze the contour of the histogram [21]. It iteratively divides a curve into a set of line segments denoted by a set of vertices (see Figure 3). If we detect no local minima between two local maxima in an image slice, we determine that the histogram of the image is unimodal; thus the image does not contain any chromosomes (see Figure $3(\mathrm{a})$ ). When a local minima is detected, we find the threshold by finding the local minima $(k)$ with the highest peakiness [21]. The peakiness is $\min (\mathrm{H}(i), \mathrm{H}(j)) / \mathrm{H}(k)$, where $i$ and $j$ are the intensity values of the neighboring local maxima and $\mathrm{H}(x)$ is the histogram value at the intensity of $x$.

\subsection{Results of region segmentation}

In Figure 3, no local minima is present on the histogram for slice 9 (Figure 3(a)), while one local maxima is found at intensity 37 corresponding to background as annotated in Figure 3, thus indicating that the histogram for slice 9 is unimodal. After applying polyline splitting to the histogram contour of the entire sequence, slices with unimodal histogram are excluded from the subsequent processes

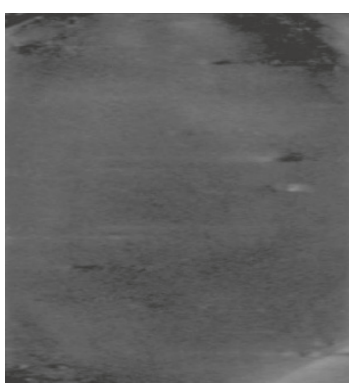

(a) Slice 2

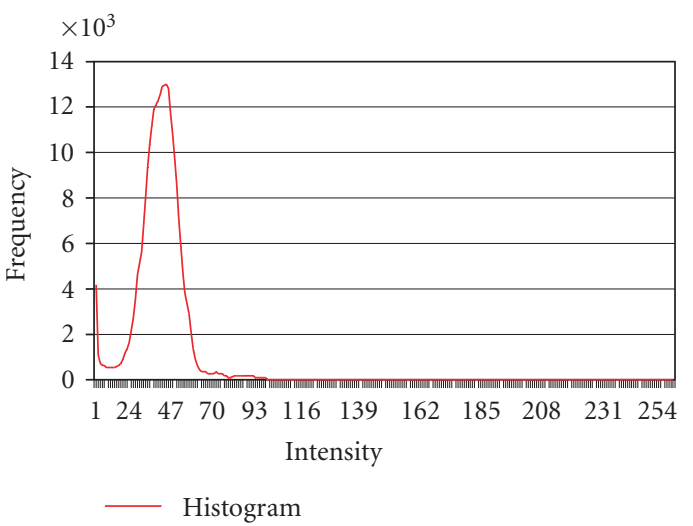

(c) Histogram of slice 2

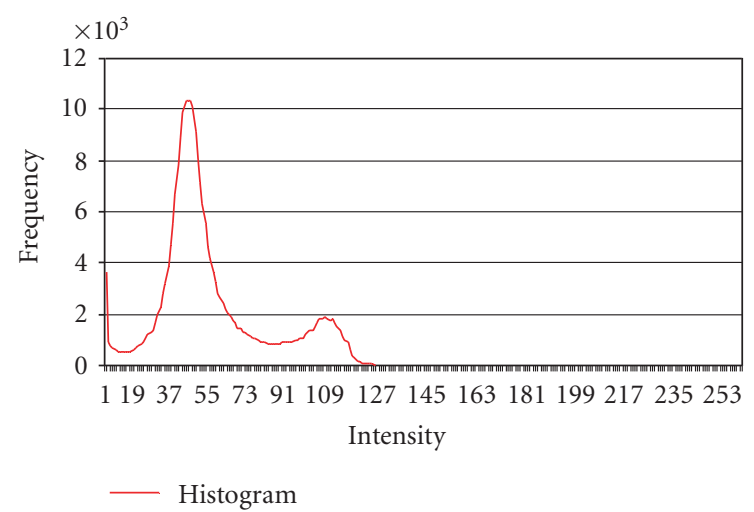

(d) Histogram of slice 35

FIgURE 2: (a) Slice 2 is one of the earlier slices of the CT scan images with a unimodal histogram distribution of intensity. (c) The histogram for slice 2. (b) Slice 35 is one of the middle slices with a bimodal histogram distribution of intensity suitable for thresholding. (d) The histogram for slice 35.

performed for visualization. They include slices 1 through 6, 9, 13 through 22, and 54 through 65 .

Figure 3(b) shows local maxima 1 and local maxima 2 found with a local minima containing the highest peakiness value in between these two local maxima. The intensity of this local minima is selected, and this value is used for region segmentation throughout the process of thresholding. A polyline distance threshold value of 25 enables proper distribution of vertices in the polyline, giving rise to a single minima vertex placed in between two local maxima vertices. 


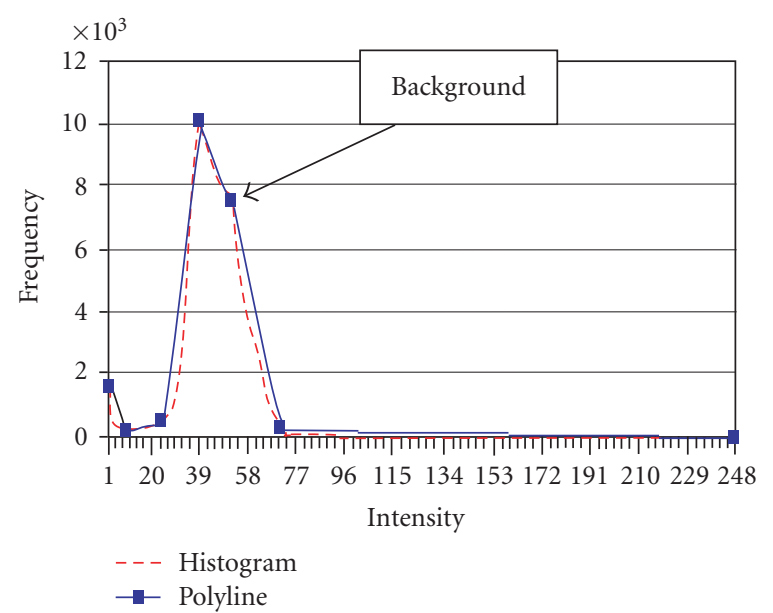

(a)

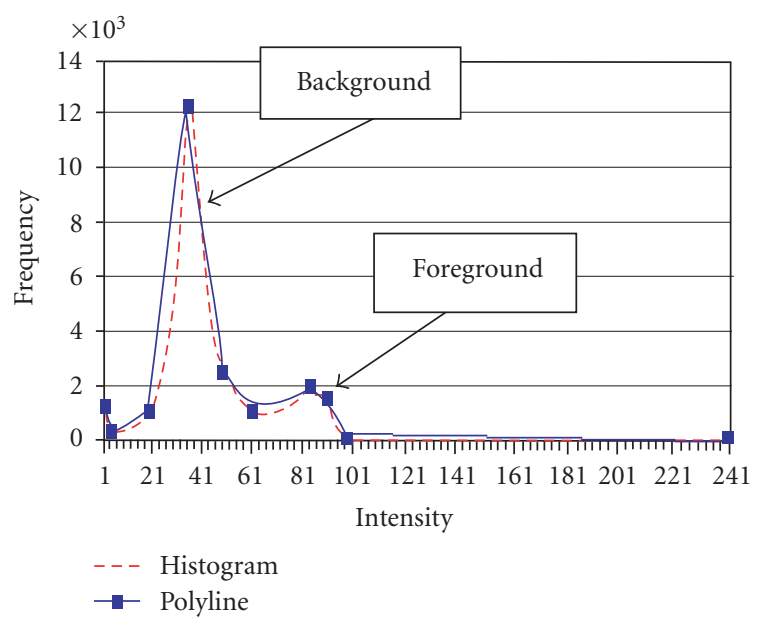

(b)

FIgURE 3: (a) Histogram for slice 9 with polyline (solid) modeling the contour of the unimodal histogram curve (dashed). (b) Histogram for slice 30 with polyline (solid) modeling the histogram contour of the bimodal histogram curve (dashed).

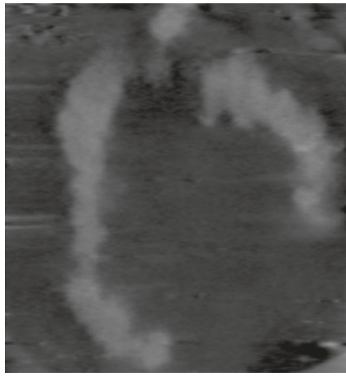

(a) Slice 30

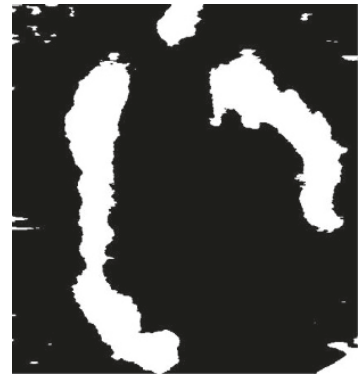

(b) Threshold slice 30 at intensity 58

FIgURE 4: (a) Slice 30 in its original form, and (b) binary thresholded image transformation of slice 30 to reveal white (foreground) chromosome regions, and black background.

Figure 4 represents the results of polyline splitting of the histogram contour, applied to the original image (Figure $4(\mathrm{a})$ ) with the threshold point at intensity 58. Thresholding then extracts the foreground chromosome objects (white represents intensity 255) from the image, and the rest is labeled as background (black is intensity 0). Table 1 shows results for every fifth slice followed by threshold values and peakiness estimates.

\subsection{Noise removal}

After region segmentation, small region removal is performed to eliminate noise in the thresholded image. Regions are detected by using connected component labeling [21], and the regions smaller than 500 pixels are removed. Note that 500 pixels is a very small region, and candidate chromosome regions are much larger in size. An appropriate value of the threshold for a given magnification level and imaged chromosome is a function of data acquisition quality,

TABLE 1: The computed threshold for every fifth slice is shown. Note that a threshold is not found for slices with the maximum peakiness of 0 , indicating that those histograms are unimodal.

\begin{tabular}{ccc}
\hline Slice & Peakiness & Threshold \\
\hline 1 & 0.0 & Not found \\
5 & 0.0 & Not found \\
10 & 1.614 & 73 \\
15 & 0.0 & Not found \\
20 & 0.0 & Not found \\
25 & 1.358 & 59 \\
30 & 1.762 & 58 \\
35 & 2.054 & 62 \\
40 & 2.005 & 58 \\
45 & 1.764 & 59 \\
50 & 1.527 & 64 \\
55 & 0.0 & Not found \\
60 & 0.0 & Not found \\
65 & 0.0 & Not found \\
\hline
\end{tabular}

magnification, and the chromosome size. For convenience, it can be easily controlled by the user. Hence, given the experimental setup such as magnification and data quality settings of the CT device during volumetric data capture, our empirically determined threshold parameter for noise removal can be adapted effectively for other data sets containing chromosomes as well. The threshold is the same for all images in the dataset; there are no separate adjustments made for each image.

\section{2D CONTOUR REFINEMENT}

\subsection{Overview}

The objective of this step is to refine and correct the contour of the chromosome regions in each $2 \mathrm{D}$ slice of the volume data recovered after segmentation and noise removal. This 


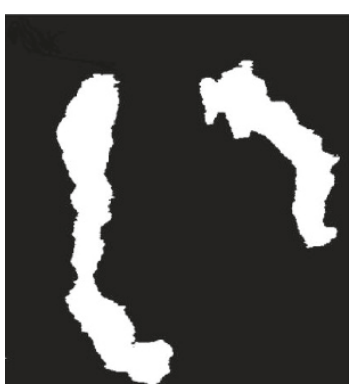

(a)

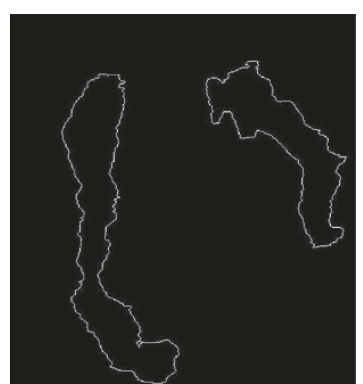

(b)
Figure 5: A slice before and after contour extraction.

step is done prior to region correspondence, so that we may first obtain an accurate contour of the chromosome regions in each $2 \mathrm{D}$ slice. The process employs $2 \mathrm{D}$ snakes to refine the contour in each slice. A snake, also known as an active contour model, builds a controlled continuity spline governed by an energy function under the influence of image and external constraint forces. The snake can be either closed or open. To obtain a desired contour, the snake is moved to a minimal energy condition. The total energy can be described as

$$
E=\int_{0}^{1} E_{\text {int }}(v(s))+E_{\text {image }}(v(s))+E_{\text {con }}(v(s)) d s,
$$

where $v(s)=(x(s), y(s)), E_{\text {int }}$ indicates the internal force of the spline due to bending, $E_{\text {image }}$ denotes the image influence, and $E_{\text {con }}$ represents the external constraint forces which permit to control a snake interactively. The threshold value and initial contour obtained after region segmentation and noise removal is used as an initial estimate for the contour refinement process. The image influence $E_{\text {image }}$ is obtained based on the image gradient computed on the Gaussian smoothed image. An example of the initial contour of the snake obtained through the process of chain coding [21] after the region segmentation and noise removal steps is shown in Figure 5.

Several different energy functions have been developed, as well as the calculation of each force term. Wei et al. proposed a novel snake algorithm based on a gradient vector flow generated through nonlinear diffusion. The advantage of this algorithm was that it was faster than other traditional approaches [27]. In this paper, snake implementation is based on [28] due to speed and stability considerations. A brief explanation of this method is included below.

\subsection{Internal force}

To describe the bending effect, two terms are considered:

$$
E_{\text {int }}=\frac{\alpha(s)\left|v_{s}(s)\right|^{2}+\beta(s)\left|v_{s s}(s)\right|^{2}}{2} .
$$

The first-order term controlled by $\alpha$ (s) causes the snake to act like a membrane, and the second-order term controlled by $\beta(s)$ makes it act like a thin plate. This modifies the behavior of a snake to be more membrane-like or thin-plate-like by adjusting these two coefficients. Notice that when $\beta(s)=0$, it represents a corner.

If $v_{i}=\left(x_{i}, y_{i}\right)$ is a node of snake, then the first-order term can be calculated easily by a backward finite-difference approximation:

$$
\left|\frac{d^{2} v_{i}}{d s^{2}}\right|^{2} \approx\left|v_{i}-v_{i-1}\right|^{2}=\left(x_{i}-x_{i-1}\right)^{2}+\left(y_{i}-y_{i-1}\right)^{2} .
$$

The second-order term represents the curvature in $v_{i}\left(x_{i}, y_{i}\right)$, which can be computed as

$$
\left|\frac{d^{2} v_{i}}{d s^{2}}\right|^{2} \approx\left(\frac{\Delta x_{i}}{\Delta s_{i}}-\frac{\Delta x_{i+1}}{\Delta s_{i+1}}\right)^{2}+\left(\frac{\Delta y_{i}}{\Delta s_{i}}-\frac{\Delta y_{i+1}}{\Delta s_{i+1}}\right)^{2},
$$

where

$$
\begin{aligned}
\Delta x_{i} & =x_{i}-x_{i-1}, \\
\Delta s_{i} & =\sqrt{\left(x_{i}-x_{i-1}\right)^{2}+\left(y_{i}-y_{i-1}\right)^{2}} .
\end{aligned}
$$

More details and discussion can be found in [28].

\subsection{Image force}

This term can provide a force that attracts the snake toward the desired feature of the object. In [22], the authors present three different force functions, those attracting a snake to lines, edges, and terminations. In this research, edge function is chosen to be the image force function. The energy function becomes

$$
E=\int\left(\alpha(s) E_{\text {cont }}+\beta(s) E_{\text {curv }}+\gamma(s) E_{\text {image }}\right) d s,
$$

where $E_{\text {cont }}$ and $E_{\text {curv }}$ are the first- and second-order terms of internal forces described in (1), respectively. As previously, one can adjust the weight of this term by changing the value of $\gamma$. In this research, $\gamma$ is set to 0.5 as empirically determined to be the most optimal setting.

\subsection{Greedy algorithm}

Once a snake and its energy function are decided, a greedy algorithm is used to find the best contour that has minimal energy.

The algorithm can be outlined as follows.

(A) For each node of the snake, evaluate the total energy at its neighbor point ( 8 points) then move each node to its neighbor point that has minimal energy. 1tem[(B)] After finding the minimal energy for all nodes, corners are checked. If a node satisfies conditions below, it could be a corner, which sets $\beta$ to 0 . This means that the curvature constraint is off.
(a) Curvature is larger than for neighbor nodes.
(b) Curvature is larger than threshold.
(c) Edge strength is above threshold.

Repeat steps (a) and (b) until the snake does not move anymore, or the number of moved nodes is below a threshold. In this research, if the moved node is less than $5 \%$ of total nodes or the iteration is more than 1000 , then we consider that the snake is in stable condition and has converged. 


\subsection{Results of $2 D$ contour refinement}

The initial contour of a snake is estimated by performing chain coding [22] after noise removal and Gaussian smoothing for an improved image energy computation. An example is shown in Figure 5. Since a single particular energy function is not suitable for every domain, combinations of the coefficients have been tested to achieve results. An empirically selected set of parameters produces the results shown in Figure 6. Through zooming (Figure 6), we can find that the snake nodes are attracted by the edges successfully, and a more accurate contour is obtained.

Our contour refinement does more of "correcting" the imperfection of boundary detection from the thresholding step rather than "processing" (such as smoothing the boundary) the boundary as shown in a sample output in Figures 7 and 8. If the initial threshold estimate is incorrect, then the algorithm does not perform optimally, and improper node placement can result. Figure 7 is extracted from the same slice as the one in Figure 6, but with a lower initial threshold estimate from the region segmentation step. The detected edges in Figure 7 have moved slightly away from the center when compared to Figure 6. The order of this snake is marked from 1 to 5 . When the snake starts moving, the node marked 3 could move to a higher edge or a lower edge. If node 3 moves in between 1 and 2, the order of the snake points becomes incorrect. Then, node 4 tends to follow node 3 due to the continuity force. Also, node 5 will follow node 4 , and so on. The method used in this research to avoid such problems checks for nodes at conflicting positions. If so, one of the nodes will be deleted. For example, if node 3 moves to node 1 , then node 3 will be deleted, and the order becomes 1 , 2,4 , and 5 . In the meantime, it is quite likely that node 4 will move to node 2's position because they have the same internal energy. If this does happen, node 4 is deleted as well. The order now becomes 1, 2, and 5. The order is now correct but the number of nodes has decreased. This may occur in various parts of a snake, which would become shorter and would reform again trying to achieve an even node distribution.

One possible resulting problem is the occurrence of gaps, such as that between nodes 1 and 2, shown in Figure 8, once the snake has reached a stable state. The reason node 2 does not move closer to position 3 is as follows. For node 2, the energies in position 3 and its current position are almost the same. Therefore, a snake may not be attracted to a very sharp point. As in most filtering techniques that remove noise, some signal may also be slightly altered. It is currently difficult to avoid the possible removal of tiny but perhaps biologically meaningful features by our contour refinement process without an extensive evaluation, which is problematical to achieve with such limited availability of datasets. However, the visualization of the contour refinement steps in Figure 6 indicates that our approach preserves the shape of the actual contour including small biologically meaningful features.

\section{REGION CORRESPONDENCE}

The objective of this step is to extract $2 \mathrm{D}$ foreground regions of the same chromosome, which are corresponded by

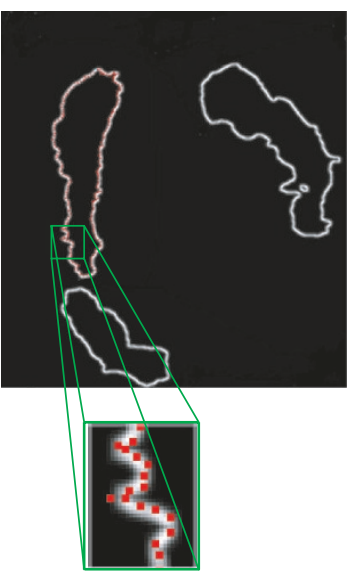

(a)

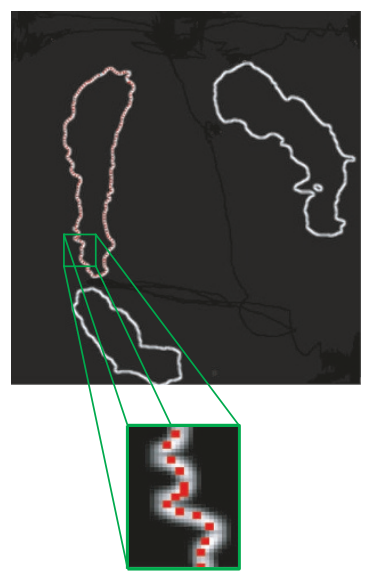

(b)
FIgURE 6: (a) Initial snake position, and (b) snake in a stable position with minimal energy. Enlarged images show the detailed changes of the snake.

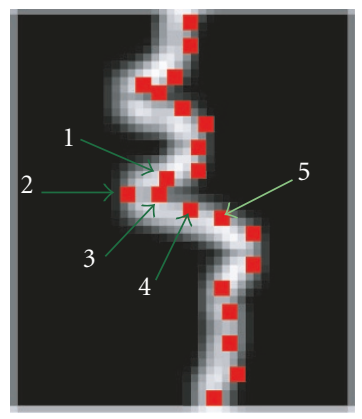

FIGURE 7: An initial position of a snake in an image with an incorrect initial threshold selection (the same slice as in Figure 6).

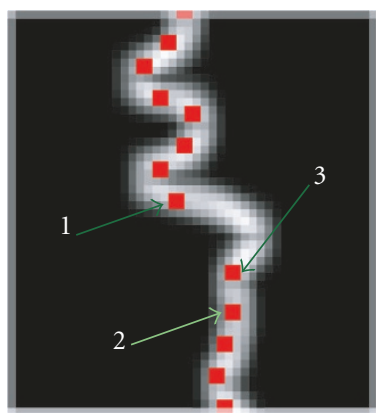

FIgUre 8: A snake in a stable position with a gap.

comparing adjacent slices, and to recover the set of regions comprising a $3 \mathrm{D}$ structure. During the previous step of $2 \mathrm{D}$ contour refinement, a more accurate contour of the chromosome regions in 2D is established after segmentation and noise removal. The $3 \mathrm{D}$ structure is produced by a set of $2 \mathrm{D}$ regions of same chromosome. We establish the correspondence between the slices by using the region comparison scheme proposed by Hoover et al. [23] that was used for 


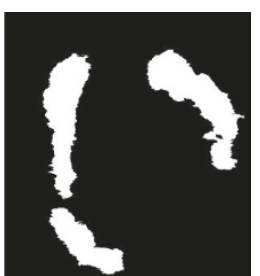

(a) Slice 23

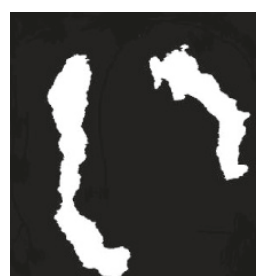

(b) Slice 38

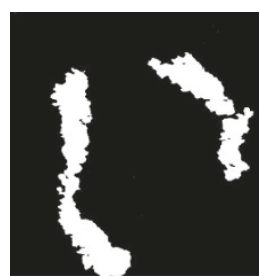

(c) Slice 53
FIgURE 9: Corresponding regions among slices. Note that the two chromosomes shown as two regions in slice 38 could be broken up in slice 23 and slice 53 .

range image segmentation comparison. Given a pair of images with segmented regions, the scheme classifies each region into five categories of correct, missed, noise, over- and under-segmentation. After the noise removal and 2D contour refinement, pairs of images are considered for regions correspondence learning. As the process iterates through the candidate pairs in the sequence, regions corresponding to 2D chromosome structure in each slice are tracked, producing sets of regions corresponding to a 3D chromosome.

Figure 9 represents two instances of segmented images from end slices (the left image is from slice 23, and the right one is from slice 53) with disjoint regions in chromosomes (chromosomes 1 and 2, resp.). The image in the middle is slice 38 after segmentation, which is a mid-slice in the sequence with both chromosomes well-defined.

The classification of regions between adjacent volume image slices allows us to perform correspondence of regions belonging to the identical or same chromosome. Among the five different types of classifications [23], namely, correct, over-segmentation, under-segmentation, missed, and noise, we are interested primarily in the correct, over- and undersegmented regions, as it is highly unlikely that the corresponded chromosome could be classified as missed or in noise regions. Metrics are designated as follows.

(i) The classification of correct is used to correspond a case of one-to-one matching of chromosome regions in adjacent slices. An instance of correct classification is specified when a pair of regions in the adjacent images has at least $\mathbf{T}$ percent of the pixels in the chromosome region $R_{i}$ in the first image marked as pixels in chromosome region $R_{j}$ in second image. This follows from the premise that $R_{i} \cap R_{j} \neq 0$ for all $i$ and $j$, if $i=j$.

(ii) The classification of over-segmentation is used to correspond a case of one-to-many maching of chromosome regions in adjacent slices. An instance of over-segmentation classification is specified when a chromosome region $R_{i}$ in the first image and a set of regions in the second image $R_{j}^{1}$ to $R_{j}^{n}$, have at least $\mathbf{T}$ percent of the pixels in chromosome region $R_{i}$ in the first image marked as pixels in the union of chromosome regions $R_{j}^{1}$ to $R_{j}^{n}$ of the second image.

(iii) The classification of under-segmentation is used to correspond a case of many-to-one matching of chromosome regions in adjacent slices. An instance of under-segmentation classification is specified when a set of chromosome regions

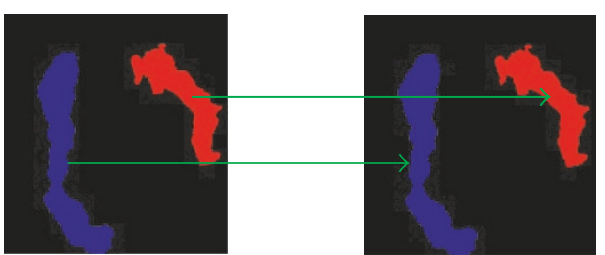

(a) Correct segmentation between slice 37 (left) and slice 38 (right)

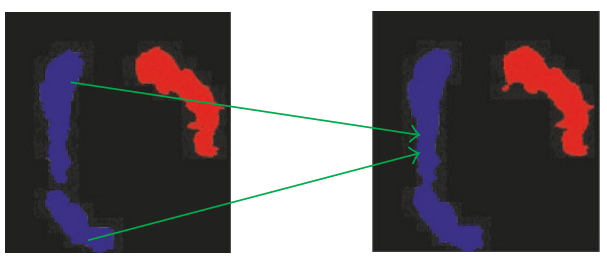

(b) Under-segmentation of region of slice 24 (left) in slice 25 (right)

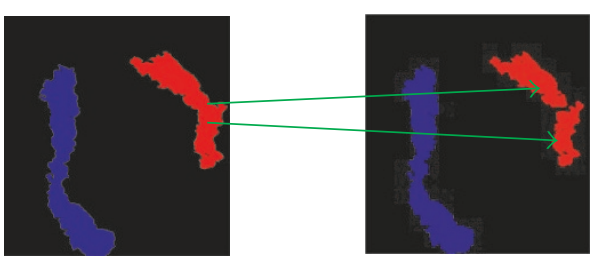

(c) Over-segmentation of region of slice 52 (left) in slice 53 (right)

FIgURE 10: Results depicting (a) correct, (b) under-, and (c) oversegmentation. All of the instances have been reclassified as either chromosome 1 (left) or 2 (right). Arrows indicate correspondences.

in the first image $R_{i}^{1}$ to $R_{i}^{n}$ and a chromosome region $R_{j}$ in the second image have at least T percent of the pixels in chromosome regions $R_{i}^{1}$ to $R_{i}^{n}$ in the first image marked as pixels in the chromosome region $R_{j}$ in the second image.

In this research, $\mathbf{T}$ is set to 250 pixels. We found that a low $\mathbf{T}$ is sufficient to determine corresponding regions of adjacent slices as belonging to the same chromosome, as the distance between adjacent slices is very small. Applying the metric described above allows us to correspond chromosome regions among adjacent slices through the sequence of volume image slices and allows recovery of sets of chromosome regions belonging to a $3 \mathrm{D}$ chromosome. Two resulting sets of regions corresponding to both chromosomes are shown in Figure 10 (left and right). When an instance of over-segmentation is detected among the adjacent slices, we are able to correspond the disjoint regions of a chromosome in one image as belonging to a single region in a different image. This method allows us to identify disjoint regions in one image as belonging to a single region in another image by classifying the observed instance as under-segmentation.

This method is used to recover regions in images that correspond to chromosomes 1 (left) and 2 (right), respectively. Figure 10 shows color images of slices representing examples of correct, under-, and over-segmentation. 


\section{3D VISUALIZATION}

\subsection{Overview}

The objective of this stage is to extract points corresponding to the contour of each chromosome object and visualize the point clouds in 3D. Such contour points are collected from each $2 \mathrm{D}$ slice to build a set of 3D point clouds. Subsequently, a $3 \mathrm{D}$ point-cloud-meshing method is applied to each set to reconstruct the surface mesh of each $3 \mathrm{D}$ chromosome.

\subsection{Point cloud}

A point cloud is a set of points. The points can be $2 \mathrm{D}$ or $3 \mathrm{D}$ points and also can be categorized into unorganized point clouds or structured point clouds. An unorganized point cloud is a point set that has only spatial position and no other information such as geometry, or shape. By contrast, a structured point cloud provides additional information that can be used for meshing, for example, break lines. In implementation, the algorithm dealing with unorganized point cloud usually transfers the data into structured data based on their coherence before generating the surface mesh. In this research, our data is actually a $2.5 \mathrm{D}$ point set, since the $z$ axis is the interval between two slices, which is set as a constant value (slice number $\times$ scale factor). However, we treat the data as a $3 \mathrm{D}$ unorganized point cloud. During the region segmentation step, slices without chromosomes are removed, hence only the remaining slices are considered for the $3 \mathrm{D}$ reconstruction and a subsequent renumbering. As a result, we do get gaps in the $3 \mathrm{D}$ reconstruction due to removed slices, which does not affect our goals of structure analysis and visualization.

\subsection{Meshing}

Meshing is the process of generating a consistent polygon model (mesh) from a given point set. The algorithm requires producing vertices, edges, and faces with shared vertices and edges. In many approaches, finite-element technique is used to find the optimal mesh. We classify algorithms as 2D, 2.5D, or $3 \mathrm{D}$, according to the data sets on which they operate. Usually quadrilateral or triangular meshes are generated in $2 \mathrm{D}$ and tetrahedral meshes are generated in 3D.

Remondino in 2003 proposed a fast and accurate approach to $3 \mathrm{D}$ reconstruction based on a set of $2 \mathrm{D}$ surface data of an object from multiple images [24]. This method has been used extensively in the surface meshing of point cloud data such as the data generated by our automatic methodology for chromosome reconstruction. The software package used in visualization (Points2Polys) also employs Remondino's 3D point-cloud-meshing algorithm. For detailed information on the meshing algorithm, we refer the reader to [24].

\subsection{Results of $3 D$ visualization}

To visualize the $3 \mathrm{D}$ chromosome objects, Points2Polys is used together with openGL. This method takes point clouds

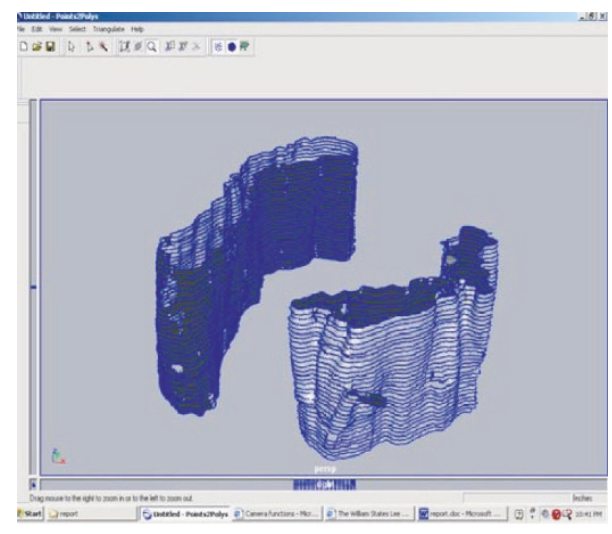

FIgure 11: View of 3D chromosome model generated by Points2Polys. Surface morphology shows invaginations, constrict points, and protrusions.

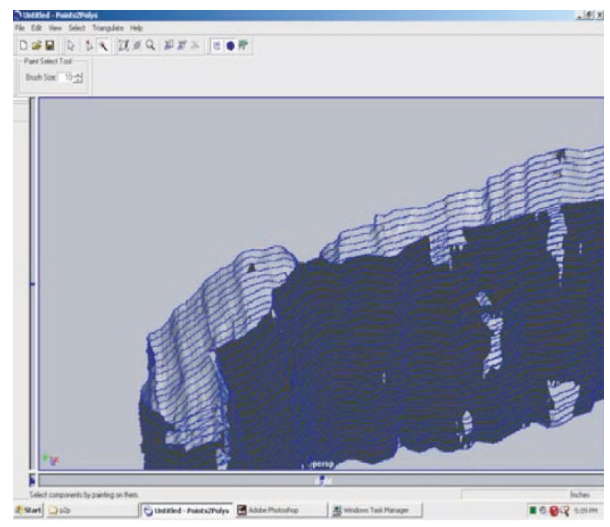

FIGURE 12: Chromosome splits up towards the top slices revealing the centromere separating the " $p$ " arm and the " $q$ " arm of the chromosome.

as input and generates triangular meshes automatically. It also provides a function that optimizes the number of points, thus requiring fewer meshes and speeding up computation and visualization. Before we import the point cloud into this software, we coded a simple module to combine all the slice nodes together, with a small $z$-interval value for distance between slices. An overview of the 3D chromosome model is shown in Figure 11. Figure 12 shows the point at which the chromosomes split up toward the end slices (an important feature selected by a biologist), which can be observed in the $3 \mathrm{D}$ model. Figure 13 shows a protrusion on the surface of the chromosome and the corresponding 2D image. This protrusion could be due to errors in segmentation or could indicate binding between an intracellular object (such as proteins or mRNA) and the chromosome. Figures 11 and 12 also reveal surface invaginations that correspond to constrict points, which can be explained by the presence of surface receptors for protein binding and signal transduction. Figure 12 also shows some well-known landmark features, including the centromere, which is the constricted region separating the " $p$ " arm and the " $q$ " arm of the chromosome. This feature 
was observed on both chromosomes upon visualization. The presence of the centromere essentially creates a dumbbelllike structure in 3D that biologists recognize as being caused by the structural conformation of DNA within the chromosome in the metaphase stage (this is also when the chromosomes are most prominently visible within the nucleus of the cell). The centromere, $p$-arm, and $q$-arm, only found in metaphase stage, are observed in our $3 \mathrm{D}$ reconstruction of the chromosomes. As our dataset of chromosomes was captured in the metaphase stage, this attests to the accuracy of our methodology to recover and visualize specific biological features of chromosomes such as the centromere, $p$-arm, $q$ arm, constrict points, receptors, and invaginations.

An inconsistent surface generated from a missed initial guess for the $2 \mathrm{D}$ contour during the refinement process results in a hole in the 3D model. These holes can be filled by changing parameters in the meshing algorithm to accommodate distant vertices in the meshing process.

From Figures 11, 12, and 13(a), one can recognize the relationship between each $2 \mathrm{D}$ cross-section through $3 \mathrm{D}$ visualization. The triangular mesh model can be visualized using openGL to add 3D manipulation functionality (translation, rotation, scaling) as well as simulating various lighting conditions to view details on the surface of a chromosome. Snapshots of chromosome visualization are provided in Figure 14, where ambient lighting at different colors and orientations is added to the scene, and a 3D chromosome surface model is viewed at various orientations in different modes (wireframe/normal).

\section{CONCLUSIONS}

In this paper, we provide a methodology for an automatic recovery and visualization of a $3 \mathrm{D}$ chromosome structure from a sequence of 2D tomographic reconstruction images taken through the nucleus of a cell. Structure is very important for biologists, as it affects chromosome functions, behavior, and the state of the cell. Chromosome analysis is significant in detection of diseases and in monitoring environmental gene mutations. The algorithm incorporates thresholding based on a histogram analysis with a polyline splitting algorithm, shape analysis, and noise removal, contour extraction via active contours, and detection of a 3D chromosome structure by establishing corresponding regions throughout the slices. Visualization using point-cloud meshing generates a $3 \mathrm{D}$ surface with a computationally inexpensive and fast approach. The 3D triangular mesh of the chromosomes provides surface detail and allows a user to interactively analyze chromosomes using visualization software. As a result, a biologist was able to localize several important features, including protrusions and shape fragmentations.

The ability to capture small biologically relevant features that are only found on chromosomes such as constric points (that may correspond to receptor sites for binding of chromosomes with adjacent intracellular organelles), protrusions, invaginations, centromere, $p$-arm, and $q$-arm attests to the accuracy and resolution of our method. The capacity to study the $3 \mathrm{D}$ geometry of chromosome struc-

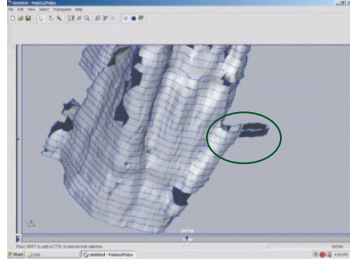

(a)

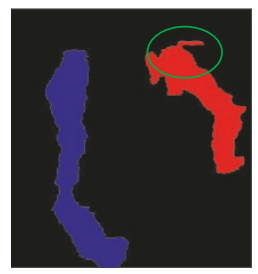

(c)

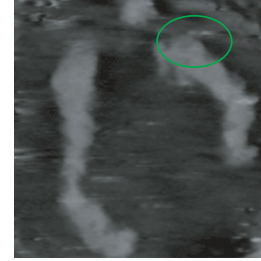

(b)

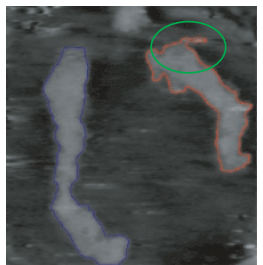

(d)
Figure 13: (a) A protruding inconsistent surface which may correspond to a surface interaction with an elongated protein molecule such as a matrix-binding proteins (anchor proteins) or a piece of mRNA. (b) Original volume image slice showing the surface artifact in 2D. (c) Initial chromosome objects after region segmentation and noise removal with the surface artifact. (d) Refined contours using snakes.

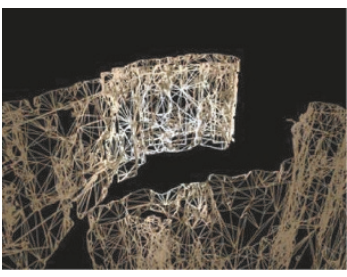

(a)

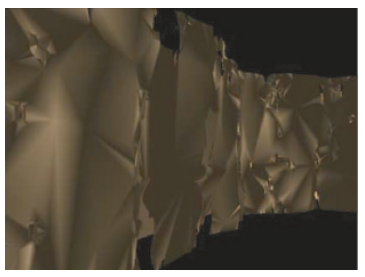

(b)

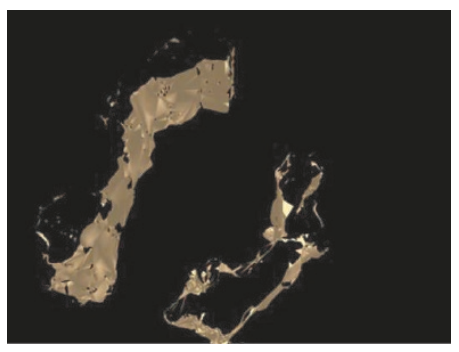

(c)

FIGURE 14: (a) Chromosomes in ambient and spot lighting in wireframe (mesh) mode. (b) The surface of chromosome 1, including surface detail and light reflection.

tures in an interactive environment is a great asset to physicians and scientists in the diagnosis and treatment of chromosomal abnormalities and the scientific analysis of surface structures of chromosomes such as binding sites with adjacent organelles or intracellular molecules such as proteins or drugs. One of the foremost advantages of our technique is the robustness of visualization based on a fairly small set of 
input images. Our automatic methodology can be extended to other common types of fluorescence data such as the ones generated from imaging modalities including electron microscopy and position emission tomography. In future work, we would like to apply our methodology to finer resolution sequences of chromosome data when they become available.

\section{ACKNOWLEDGMENTS}

We wish to thank Professor John W. Sedat and his laboratory colleagues at the University of California San Francisco for the data, and Dr. William Moss (LLNL) for discussing the problem with us. This work was performed under the auspices of the US Department of Energy by University of California Lawrence Livermore National Laboratory under Contract no. W-7405-Eng-48. UCRL-PROC-203893.

\section{REFERENCES}

[1] R. J. Gardner, Geometric Tomography, Cambridge University Press, Cambridge, UK, 1995.

[2] B. Lerner, H. Guterman, and I. Dinstein, "A classificationdriven partially occluded object segmentation (CPOOS) method with application to chromosome analysis," IEEE Transactions on Signal Processing, vol. 46, no. 10, pp. 28412847, 1998.

[3] H. Shi, P. Gader, and H. Li, "Parallel mesh algorithm for grid graph shortest paths with application to separation of touching chromosomes," The Journal of Supercomputing, vol. 12, no. 1-2, pp. 69-83, 1996.

[4] B. Lerner, M. Levinstein, B. Rosenberg, H. Guterman, L. Dinstein, and Y. Romem, "Feature selection and chromosome classification using a multilayer perceptron neural network," in Proceedings of IEEE International Conference on Neural Networks, vol. 6, pp. 3540-3545, Orlando, Fla, USA, June-July 1994.

[5] E. Vidal and M. J. Castro, "Classification of banded chromosomes using error-correcting grammatical interface (ECGI) and multilayer perceptron (MLP)," in VII National Symposium on Pattern Recognition and Image Analysis, pp. 31-36, 1997.

[6] J. M. Keller, P. Gader, O. Sjahputera, C. W. Caldwell, and H.M. T. Huang, "A fuzzy logic rule-based system for chromosome recognition," in Proceedings of the 8th IEEE Symposium on Computer-Based Medical Systems, pp. 135-132, Lubbock, Tex, USA, June 1995.

[7] C. Imelinska, M. S. Downes, and W. Yuan, "Semi-automatic color segmentation of anatomical tissue," Computerized Medical Imaging and Graphics, vol. 24, no. 3, pp. 173-180, 2000.

[8] M. Holden, E. Steen, and A. Lundervold, "Segmentation and visualization of brain lesions in multispectral magnetic resonance images," Computerized Medical Imaging and Graphics, vol. 19, no. 2, pp. 171-183, 1995.

[9] J. Yan, T.-G. Zhuang, L. H. Schwartz, and B. Zhou, "Lymph node segmentation from CT images using fast marching method," Computerized Medical Imaging and Graphics, vol. 28, no. 1-2, pp. 33-38, 2004.

[10] H. J. Noordmans and A. W. M. Smeulders, "Detection and characterization of isolated and overlapping spots," Computer Vision and Image Understanding, vol. 70, no. 1, pp. 23-35, 1998.

[11] Z. Qingsong, K. C. Keong, and N. W. Sing, "Interactive surgical planning using context based volume visualization tech- niques," in Proceedings of IEEE International Conference on Information Visualization 2002, pp. 323-330, November 2002.

[12] R. A. Banvard, "The visible human project $(\mathbb{R}$ image data set from inception to completion and beyond," in Proceedings of CODATA 2002: Frontiers of Scientific and Technical Data, Montral, Canada, September-October 2002.

[13] K. R. Subramanian, M. J. Thubrikar, B. Fowler, M. T. Mostafavi, and M. W. Funk, "Accurate 3D reconstruction of complex blood vessel geometries from intravascular ultrasound images: in vitro study," Journal of Medical Engineering and Technology, vol. 24, no. 4, pp. 131-140, 2000.

[14] M. R. Arnison, C. J. Cogswell, N. I. Smith, P. W. Fekete, and K. G. Larkin, "Using Hilbert transforms for 3D visualization of differential interference contrast microscope images," Journal of Microscopy, vol. 199, no. 1, pp. 79-84, 2000.

[15] P. Engelhardt, J. Ruokolainen, A. Dulenc, L. G. Överstedt, H. Mehlin, and U. Skoglund, "3D-reconstruction by electron tomography (EMT) of whole-mounted DNA-depleted metaphase chromosomes show scaffolding macro coils, 30nm fibers and 30-nm particles," in Proceedings of International Conference on 3D Image Processing in Microscopy, Munich, Germany, April 1994.

[16] J. Liu, J. K. Udupa, D. Odhner, D. Hackney, and G. Moonis, "A system for brain tumor volume estimation via MR imaging and fuzzy connectedness," Computerized Medical Imaging and Graphics, vol. 29, no. 1, pp. 21-34, 2005.

[17] R. A. Zoroofi, Y. Sato, T. Nishii, N. Sugano, H. Yoshikawa, and S. Tamura, "Automated segmentation of necrotic femoral head from 3D MR data," Computerized Medical Imaging and Graphics, vol. 28, no. 5, pp. 267-278, 2004.

[18] M. A. Viergever, J. B. A. Maintz, W. J. Niessen, et al., "Registration, segmentation, and visualization of multimodal brain images," Computerized Medical Imaging and Graphics, vol. 25, no. 2, pp. 147-151, 2001.

[19] D. Levin, U. Aladl, G. Germano, and P. Slomka, “Techniques for efficient, real-time, $3 \mathrm{D}$ visualization of multi-modality cardiac data using consumer graphics hardware," Computerized Medical Imaging and Graphics, vol. 29, no. 6, pp. 463-475, 2005.

[20] P. Shaw, D. Agard, Y. Hiraoka, and J. Sedat, “Tilted view reconstruction in optical microscopy: three-dimensional reconstruction of Drosophila melanogaster embryo nuclei," Biophysical Journal, vol. 55, no. 1, pp. 101-110, 1989.

[21] R. Jain, R. Kasturi, and B. G. Schunck, Machine Vision, MIT Press and McGraw-Hill, Boston, Mass, USA, 1995.

[22] M. Kass, A. Witkin, and D. Terzopoulos, "Snake: actve contour models," in Proceedings of 1st International Conference on Computer Vision, pp. 259-269, 1987.

[23] A. Hoover, G. Jean-Baptiste, X. Jiang, et al., "An experimental comparison of range image segmentation algorithms," IEEE Transactions on Pattern Analysis and Machine Intelligence, vol. 18, no. 7, pp. 673-689, 1996.

[24] F. Remondino, "From point cloud to surface: the modeling and visualization problem," in Proceedings of International Archives of Photogrammetry, Remote Sensing and Spatial Information Sciences, in International Workshop on Visualization and Animation of Reality-based 3D Models, Tarasp-Vulpera, Switzerland, February 2003.

[25] T. McInerney and D. Terzopoulos, "Deformable models in medical image analysis: a survey," Medical Image Analysis, vol. 1, no. 2, pp. 91-108, 1996.

[26] F. Wilcoxon, "Individual comparisons by ranking methods," Biometrics, vol. 1, pp. 80-83, 1945. 
[27] M. Wei, Y. Zhou, and M. Wan, "A fast snake model based on non-linear diffusion for medical image segmentation," Computerized Medical Imaging and Graphics, vol. 28, no. 3, pp. 109-117, 2004.

[28] D. J. Williams and M. Shah, "A fast algorithm for active contours and curvature estimation," Image Understanding, vol. 55, no. 1, pp. 14-26, 1992.

Sabarish Babu received a B.S. degree in biology with a concentration in microbiology in 1999 and an M.S. degree in information technology with a concentration in advanced databases and knowledge discovery from the University of North Carolina at Charlotte in 2002. Currently he is a Ph.D. student in information technology focusing on virtual reality and 3D humancomputer interaction, working under the

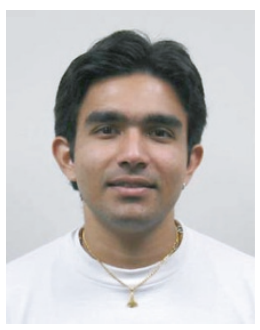
supervision of Dr. Larry F. Hodges in the Future Computing Lab (http://www.cs.uncc.edu/fcl). His research interests include computer vision, visualization, and VR for training and therapy. For more information, see http://www.cs.uncc.edu/ sbabu/.

Pao-Chuan Liao received the M.S. degree in computer science from University of North Carolina at Charlotte in 2003.

Min C. Shin received the B.S., M.S., and Ph.D. degrees in computer science from the University of South Florida, Tampa, in 1992, 1996, and 2001, respectively. He received University of South Florida Graduate Council's Outstanding Dissertation Prize. $\mathrm{He}$ is currently an Assistant Professor in the Department of Computer Science at the University of North Carolina at Charlotte. His research interests include medi-

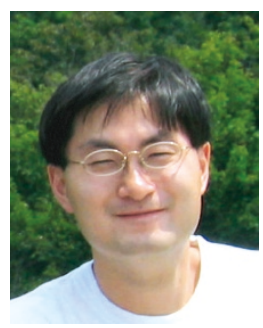
cal image analysis, gesture recognition, and skin detection evaluation. He is a Member of IEEE, UPE, and the Golden Key Honor Society. More information can be obtained from http://www.cs. uncc.edu/ $\sim$ mcshin/.

Leonid V. Tsap received the M.S. and Ph.D. degrees in computer science from the University of South Florida, Tampa, in 1995 and 1999, respectively. He is a recipient of the University of South Florida Graduate Council's Outstanding Dissertation Prize and Provost's Commendation for Outstanding Teaching by a Graduate Student. He is currently with the Systems Research Group at the University of Califor-

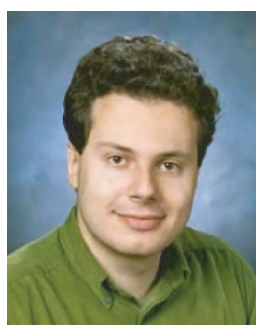
nia Lawrence Livermore National Laboratory. He is a Member of the IEEE-CS and ACM. He is a Member of the Editorial Board of the Pattern Recognition journal, and of the Program Committee of IEEE Workshop on Articulated and Nonrigid Motion held in conjunction with CVPR '04. His current research interests include applying dynamic self-adapting models to complex evolving data analysis in bioinformatics, nanoscale analysis, medical diagnostics, perceptual interfaces, computer vision, intelligent biometrics, security, communications, and other areas. His research resulted in more than 30 refereed publications. More information can be obtained from http://marathon. csee.usf.edu/ tsap and http://www.llnl.gov/CASC/people/tsap. 